\title{
NATURAL CONVECTION THROUGH VERTICAL POROUS STRATUM
}

\author{
N. RUDRAIAH \\ Department of Mathematics, Central College, Bangalore University, Bangalore, India \\ and \\ S. T. NAGARAJ \\ Department of Physics, University of Agricultural Sciences, Hebbal, Bangalore, India
}

(Communicated by S. I. PAI)

\begin{abstract}
The combined effect of Darcy and viscous resistances on the fully developed natural convection of a fluid between two heated vertical plates is investigated. When Darcy and viscous dissipations, in the energy equation, are negligible the energy and momentum equations become decoupled and we obtain the modified Poiseuille flow distribution through porous media. The deviation of the velocity and temperature distributions from those existing in modified Poiseuille flow are presented for various porous number $\sigma$ $(=b / k, b$ is the spacing between the plates and $k$ is the permeability of the porous medium) when dissipations are not neglected. It is shown that the increase in porous number rapidly decreases the influence of both viscous and Darcy dissipations on velocity and temperature profiles. Therefore, by suitable adjustment of dissipation terms, it is possible to control the temperature distribution which will be of some use in plant physiology.
\end{abstract}

\section{INTRODUCTION}

THE STUDY of the effect of convection on the flow and heat transfer processes through a porous medium plays an important role in agricultural engineering and throws some light on the influence of environment like temperature and pressure on the germination of seeds. It is also of interest in petroleum industry in extracting pure petrol from the crude. The study of flow through porous media is also of importance in soil physics and geohydrology.

Studies associated with such flows were based on the Darcy's empirical equation

$$
\mathbf{q}=-\frac{\text { const }}{\mu} \nabla p
$$

where $q$ is the mean filter velocity; $\mu$ is the viscosity of the fluid; $\nabla p$ is the pressure gradient.

Later Muskat [1] has shown that this constant in eqn (1.1) must depend on the permeability of the porous material and showed that

$$
\mathbf{q}=-\frac{k}{\mu} \nabla p
$$

where $k$ is the permeability of the porous material and has the dimensions of length squared. This equation has been used ever since to study the dynamic behaviour of flow through porous media. Equation (1.2), which represents an irrotational flow, is the statistical average of the Navier-Stokes equations applied to flow through porous media and is valid only when $k$ is very small. It is of interest to compare the viscous $\left(\mu \nabla^{2} q\right)$ and Darcy resistance $(\mu / k q)$ terms, i.e.

$$
\left[\frac{\mu \nabla^{2} \mathbf{q}}{(\mu / k) q}\right]=\frac{k}{d^{2}}
$$

where $d$ is the characteristic length. If we are dealing with sand as the porous material, $k \approx 10^{-6} \mathrm{~cm}^{2}$ and for $d=10^{-1} \mathrm{~cm}, k / d^{2} \approx 10^{-4}$ which is very much less than 1 . In that case viscous resistance cannot be neglected in comparison with the Darcy's resistance. In this case, following Tam [2], the velocity can be represented in the form

$$
q=-\nabla \phi+\nabla \times \mathbf{F}
$$


where the first term represents irrotational flow and the second represents rotational flow. This rotational effect is due to the effect of solid material of the porous medium on the fluid flow. Therefore, the aim of this paper is to study the flow through porous media by considering both viscous and Darcy's resistance terms.

For this purpose, the basic equations for flow through porous media are discussed in Section 2. These equations clearly bring out the effect of solid on the flow and from this we can easily obtain the usual Darcy's eqn (1.2) as a limiting case. In Section 3, these equations are applied to study the vertical flow through a porous medium by considering both forced and free convection effects and its analogy with MHD is brought out. Approximate solutions to the coupled non-linear equations are derived for the steady flow. The velocity, mass flow rate, friction factor, temperature, skin friction and the rate of heat transfer are shown graphically followed by a qualitative discussion.

\section{BASIC EQUATIONS}

It is well known that the flow through porous media is governed by the Muskat [1] model of the Darcy's equation

$$
\mathbf{q}=\frac{k}{\mu}(-\nabla p+\rho \mathbf{g})
$$

which is valid, as described in Section 1 , when $k / d^{2}$ is very much less than unity. The aim of the present paper is to discuss the flow through porous media when $k / d^{2} \approx 1$, which is the case with the sparse distribution of a swarm of particles (see Prabhamani and Rudraiah [3]). In this case, the basic equations will depend on the viscous drag force exerted on the cloud of particles under the assumption of low Reynolds' number. Brinkman[4] has obtained this viscous drag force assuming that the force on a particle situated in the cloud of particles could be calculated as if it were a solid particle embedded in a porous mass. He represented the porous mass by modifying Stokes' equation adding Darcy resistance term to it so that the effect of all other particles is treated in an average sense and the resulting equation is a modification of Darcy's equation. Since the latter equation is empirical, Brinkman's result has not been generally regarded as a completely rigorous theoretical model to the problem even though by comparing with the experimental data the success of Brinkman's formula is indisputable.

Tam[2] put Brinkman's method in better theoretical shape by treating the swarm of particles as point forces in Stokes flow and ensemble averaging over all particle position except that of primary particle. The resulting equations for steady, incompressible flow are the same as Brinkman's and for the problem under consideration, they can be expressed as

$$
\begin{aligned}
\mu \nabla^{2} \mathbf{q}-\frac{\mu}{k} \mathbf{q} & =\nabla p-\rho \mathbf{g} \\
\nabla \cdot \mathbf{q} & =\mathbf{0} .
\end{aligned}
$$

The physical meaning of these equations can be well understood by examining their fundamental solution. If a point force of magnitude $D$ is applied at the origin inside a homogeneous, isotropic swarm of particles, then the solution of the above equations in the absence of external body force is given by (see Lamb[5])

$$
\mathbf{q}=\frac{D}{4 \pi \mu}\left[\frac{\mathrm{e}^{-r / \sqrt{ } k}}{r} \hat{e}_{x}+\frac{\nabla\left\{(\partial / \partial x) k\left(1-\mathrm{e}^{-r / \sqrt{ } k}\right)\right\}}{r}\right]=\nabla \phi+\nabla \times \mathbf{F}
$$

where $\hat{e}_{x}$ is the unit vector in the axial direction; $r$ is the distance from the origin; $\nabla \times F$ represents the transverse component of velocity; $\nabla \phi$ represents the longitudinal velocity.

These transverse velocity components are effectively confined within a distance $\sqrt{ } k$, the screening distance or the boundary layer thickness. For a distance larger than $\sqrt{ } k$, the longitudinal velocity components matter. In other words, in the boundary layer region, we have to take the equation of the form (2.2) which represents both rotational and irrotational flows, whereas outside the boundary layer region, we have to take Darcy's eqn (2.1) which represents 
only the potential flow. In this paper, we are concerned only with equations of the form (2.1) and (2.2).

In addition to the assumptions discussed earlier, we make the Boussinesq approximation which amounts to neglect of density variation except in the buoyancy term. This implies that the fluctuations in density occur principally as a result of thermal rather than pressure variations. This is a natural approximation in the case of liquid, valid when the speed of flow is much less than that of sound in the medium and when accelerations are slow compared with those associated with sound waves. In this case the equation of state is

$$
\rho=\rho_{0}\left[1-\beta\left(T-T_{0}\right)\right]
$$

where $\beta$ is the thermal expansion coefficient; $\rho_{0}$ is the density at $T=T_{0}$. The temperature $T$ is given by the energy equation

$$
C_{p} \rho_{0}(\mathbf{q} \cdot \nabla) T=K \nabla^{2} T+\Phi+\frac{\mu}{k} \mathbf{q} \cdot \mathbf{q}
$$

where $C_{p} \rho_{0}$ is the heat capacity of the fluid; $K$ is the thermal conductivity of the saturated medium; $(\mu / k) q^{2}$ is the Darcy's dissipation and

$$
\Phi=2 \mu\left[\left(\frac{\partial u}{\partial x}\right)^{2}+\left(\frac{\partial v}{\partial y}\right)^{2}+\left(\frac{\partial w}{\partial z}\right)^{2}+\frac{1}{2}\left(\frac{\partial u}{\partial y}+\frac{\partial v}{\partial z}\right)^{2}+\frac{1}{2}\left(\frac{\partial v}{\partial z}+\frac{\partial w}{\partial x}\right)^{2}+\frac{1}{2}\left(\frac{\partial w}{\partial x}+\frac{\partial u}{\partial y}\right)^{2}\right]
$$

is the viscous dissipation, and $u, v, w$ are the components of velocity in the increasing directions of $x, y$ and $z$ respectively.

\section{LAMINAR FREE CONVECTION THROUGH A VERTICAL POROUS STRATUM}

Laminar free convection through a channel without any porous medium has been studied extensively by many authors (see Sparrow et al.[6], Ostrach [7]) but the same through a porous medium has not been given much attention. Since this problem involves interaction of several phenomena, it has stimulated interest in many modern technology. Therefore, in this section we investigate the fully developed free convection in a viscous fluid flowing through a vertical stratum considering both viscous and Darcy resistances. In other words, heat is generated within the fluid by both viscous and Darcy dissipations. Attention is directed to the situation where both forced and free convection act simultaneously in establishing the flow and temperature flelds adjacent to a heated or a cooled body. The motion of the fluid is determined first by neglecting viscous and Darcy dissipations and then their effect on temperature and velocity profiles is evaluated. It is found that the permeability acts to decrease the dissipation effects. The results of this section are of interest in metal production, underground bed flow, oil recovery from partially depleted reservoirs, agricultural engineering and various applications in chemical engineering.

\subsection{Convection equations}

The physical configuration for flow through vertical porous stratum under investigation is as shown in Fig. 1. For the fully developed flow model considered, conservation of momentum given by eqn (2.2) using equation of state (2.5) requires

$$
\frac{\mathrm{d}^{2} u}{\mathrm{~d} y^{2}}-\frac{u}{k}+\frac{g \beta\left(T-T_{0}\right)}{\nu}=0
$$

where $u$ is the velocity in the $x$ direction; $T$ is the temperature field; $T_{0}$ is the ambient temperature.

Both the velocity and temperature are functions of $y$ only. For the flow model considered, the conservation of energy (2.6) for the fluid requires

$$
\frac{\mathrm{d}^{2} T}{\mathrm{~d} y^{2}}+\frac{\rho_{0} \nu}{K}\left(\frac{\mathrm{d} u}{\mathrm{~d} y}\right)^{2}+\frac{\rho_{0} \nu}{k K} u^{2}=0 .
$$




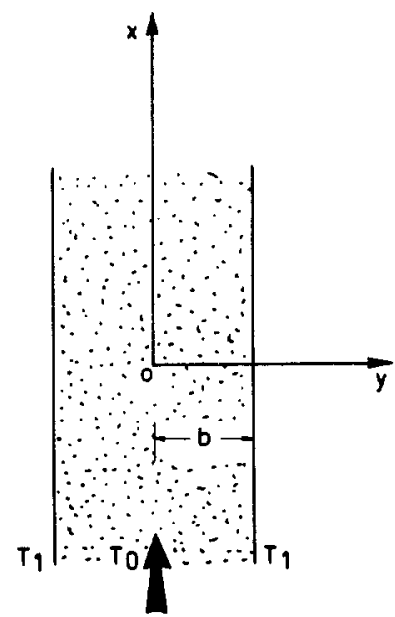

Fig. 1. Physical configuration of flow.

The second and third term on the left hand side of the eqn (3.2) represent the viscous and Darcy dissipations respectively. We note that this Darcy dissipation is similar to the ohmic dissipation in magnetohydrodynamics when magnetic Reynold's number is small.

The boundary conditions on velocity and temperature field are

$$
\begin{gathered}
u=0 \text { and } T=T_{1} \text { at } y=b \\
\frac{\mathrm{d} u}{\mathrm{~d} y}=0 \quad \text { and } \quad \frac{\mathrm{d} T}{\mathrm{~d} y}=0 \text { at } y=0 .
\end{gathered}
$$

The first boundary condition on velocity represents the no-slip condition and the second condition is obtained by the assumption of symmetry. The boundary conditions on temperature follows from the symmetry and by maintaining the walls at the same temperature $T_{1}$. The temperature difference between the walls and the fluid in the centre of the vertical stratum must be small, otherwise the flow will not be laminar. By use of the following non-dimensional quantities

$$
\begin{gathered}
Y=\frac{y}{b} \\
\theta=\frac{T-T_{0}}{T_{1}-T_{0}} \\
U=\frac{\nu}{g \beta b^{2}\left(T_{1}-T_{0}\right)} u
\end{gathered}
$$

equations (3.1) and (3.2) can be placed in the non-dimensional form yielding

$$
\begin{gathered}
\frac{d^{2} U}{d Y^{2}}-\sigma^{2} U+\theta=0 \\
\frac{d^{2} \theta}{d Y^{2}}+N\left(\frac{d U}{d Y}\right)^{2}+\sigma^{2} N U^{2}=0
\end{gathered}
$$

where $\sigma$ is the dimensionless number measuring the Darcy resistance given by

$$
\sigma=b / \sqrt{ } k
$$

and $N$ is the dimensionless number measuring the buoyancy force given by

$$
\begin{aligned}
N & =\frac{\rho_{0} g^{2} \beta^{2} b^{4}\left(T_{1}-T_{0}\right)}{K \nu} \\
& =\frac{\mu b g \beta}{K} G_{r}
\end{aligned}
$$


where

$$
G_{r}=\frac{\beta g b^{3}\left(T_{1}-T_{0}\right)}{\nu^{2}}
$$

is the Grashof number.

Thus, the boundary conditions on $U$ and $\theta$ are

$$
\begin{aligned}
U=0 \text { and } \theta=1 \quad \text { at } & Y=1 \\
\frac{\mathrm{d} U}{\mathrm{~d} Y}=0 & \text { and } \frac{\mathrm{d} \theta}{\mathrm{d} Y}=0 \text { at } Y=0 .
\end{aligned}
$$

\subsection{Solutions}

Equations (3.08) and (3.09) are non-linear because of dissipation terms and contain two unknowns $U$ and $\theta$ which must be solved simultaneously to yield the desired velocity profiles. Due to their non-linearity, eqns (3.8) and (3.9) are difficult to solve. However the fact that $N$ is small in most practical problems allows us to employ a perturbation technique to solve these non-linear equations. We can write

$$
\begin{aligned}
U & =U_{0}+N \phi \\
\theta & =\theta_{0}+N \epsilon
\end{aligned}
$$

where the zero-subscript quantities are the solutions for the case in which $N$ is equal to zero (which is equivalent to neglecting viscous and Darcy dissipations), and $\phi$ and $\epsilon$ represent the perturbation quantities relative to $U_{0}$ and $\theta_{0}$. Here the value of $N$ indicates the state of the plates. Since $N$ depends on the plates, it can take positive, zero or negative values depending on the temperature of the plates. If $T_{1}-T_{0}<0$, then the plate temperature is less than the ambient temperature and hence the convection currents flow towards the plate and in this sense the plate is heated from the externally supplied heat energy of the fluid. Hence $N<0$ corresponds to an externally heated plate. Similarly $N>0$ corresponds to external cooling of the plate and $N=0$ corresponds to the absence of the convection currents, i.e. absence of dissipation. The quantities $U_{0}$ and $\theta_{0}$ obtained by solving linear differential equations resulting by setting $N=0$ are given by

$$
\begin{aligned}
& U_{0}=\frac{1}{\sigma^{2}}\left[1-\frac{\cosh \sigma Y}{\cosh \sigma}\right] \\
& \theta_{0}=1
\end{aligned}
$$

In the limit $\sigma \rightarrow 0$, eqn (3.18) tends to the limit

$$
U_{f 0}=\frac{1}{2}\left[1-Y^{2}\right]
$$

which is the usual parabolic distribution in the channel in the absence of porous medium and bounded by impermeable plates. We note that eqn (3.18) in which $\sigma$ replaced by Hartmann number is similar to the Hartmann velocity distribution in the channel in MHD. Therefore, the velocity distribution through a porous channel is similar to the MHD flow in a channel where the kinetic energy is converted into electromagnetic energy. Equation (3.18) is numerically evaluated for different values of $\sigma$ and is shown in Fig. 2. We find that the velocity decreases with the increase in $\sigma$. This flattening effect is due to the fact that the resistance offered by the solid material on the flow is to retard the flow which is analogous to the effect of magnetic field on the flow of a conducting fluid. For possible experimental verification, the variation of the velocity is expressed in terms of the percentage change. We observe, from Fig. 2, that at $Y=0.2$, when $\sigma$ is increased from 2 to 4 , the velocity falls by $67 \%$. This percentage fall goes on decreasing slowly as $Y$ increases and rapidly as $\sigma$ increases. For example, when $\sigma$ is increased from 2 to 4 , at $Y=0$ the fall is $67 \%$, at $Y=0.2$ the fall is $66.6 \%$, at $Y=0.4$ the fall is $64.86 \%$ and so on, whereas when $\sigma$ is increased from 18 to 20 , the fall is only $19 \%$. 


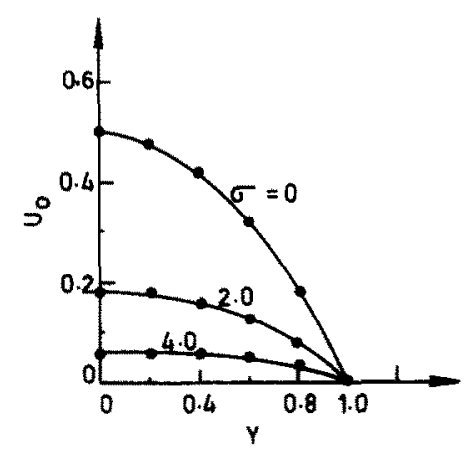

Fig. 2. Velocity profile when dissipations are neglected.

Before proceeding to find the perturbation quantities, it is of interest to find the mass flow rate and the friction factor in the absence of dissipation. The mass flow rate depicts quantitatively the effect of permeability on the flow and the friction factor gives the information as to under what Reynold's number the flow is laminar.

If $m_{p_{0}}$ denotes the half-channel flow rate per unit channel width in the absence of dissipation, for flow through the channel with porous medium, then

$$
\begin{aligned}
m_{p_{0}} & =\int_{0}^{b} \rho_{0} u_{0} \mathrm{~d} y \\
& =-\frac{\rho_{0} G b^{3}}{\sigma^{2}}\left[1-\frac{\tanh \sigma}{\sigma}\right]
\end{aligned}
$$

where

$$
G=-\frac{g \beta\left(T_{1}-T_{0}\right)}{\nu}
$$

On the other hand, for a channel without the porous material, the mass flow rate $m_{f_{0}}$ in the absence of dissipation is given by

$$
m_{f_{0}}=-\frac{\rho_{0} G b^{3}}{3}
$$

It is of interest to note that the mass flow can be stopped by setting $G=0$, i.e. $T_{\mathrm{t}}=T_{0}$.

The mass flow rates through the channel with the porous material and without the porous material may now be compared. For the conditions of equal pressure gradients and channel widths the ratio of eqns (3.23) and (3.24) yields

$$
\frac{m_{P 0}}{m_{f_{0}}}=\frac{3}{\sigma^{2}}\left[1-\frac{\tanh \sigma}{\sigma}\right]
$$

This ratio of mass flow rates is numerically evaluated and is shown in Fig. 3. From this it is evident that the mass flow rate ratio decreases as $\sigma$ increases. We also observe that when $\sigma$ is increased from 2 to 4 , there is a fall in the ratio by $63.80 \%$. This behaviour is similar to that of velocity distribution.

The above solutions are obtained under the assumption of laminar flow. It is of practical interest to know up to what Reynold's number the laminar flow is valid. This can be achieved by calculating the friction factor $C_{f}$ defined by

$$
C_{f}=-\frac{\nu G D}{\frac{1}{2} \bar{\mu}_{0}^{2}}
$$

where $D$ is the equivalent diameter ( $D=4 b$ for the channel considered in this paper). 


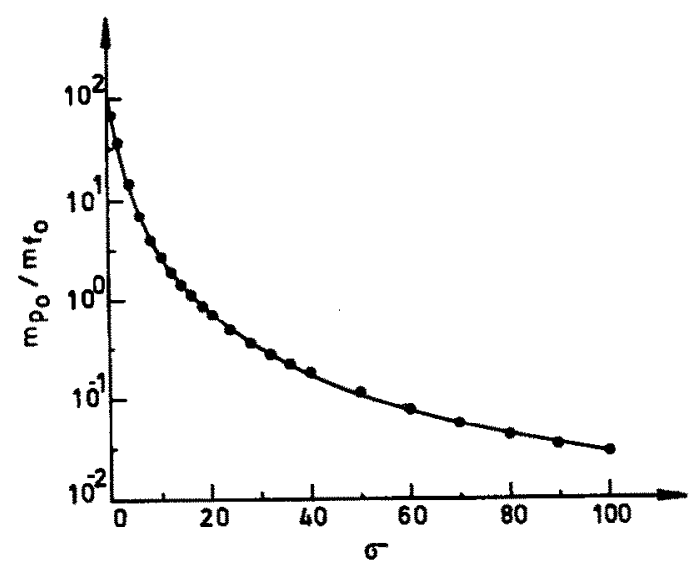

Fig. 3. Ratio of mass flow rate with and without the porous medium vs $\sigma$.

Since

$$
\bar{u}_{0}=\frac{1}{b} \int_{0}^{b} u_{0} \mathrm{~d} y=-\frac{G b^{2}}{\sigma^{2}}\left[1-\frac{\tanh \sigma}{\sigma}\right]
$$

we get

$$
C_{f}=\frac{32 \sigma^{2}}{\operatorname{Re}\left[1-\frac{\tanh \sigma}{\sigma}\right]}
$$

where

$$
\operatorname{Re}=\frac{\bar{u}_{0} D}{\nu}
$$

is the Reynold's number.

This shows that $C_{f} R_{e}$ product is constant (independent of Reynold's number) for a channel of fixed height and a given porous medium. This $C_{f} R_{e}$ product in the case of flow through a channel in the absence of porous material is given by

$$
C_{3}^{*} R_{e}^{*}=96 .
$$

Therefore

$$
\frac{C_{f} R_{e}}{C_{f}^{*} R_{e}^{*}}=\frac{\sigma^{2}}{3\left[1-\frac{\tanh \sigma}{\sigma}\right]}
$$

which is independent of Reynold's number but depends on the permeability of the medium and the width of the channel. This ratio is numerically evaluated for different values of $\sigma$ and is shown in Fig. 4. From this, it is clear that the ratio increases with increasing $\sigma$. Therefore, for large values of $\sigma$, the flow is laminar.

The above results are true only for $N=0$ which is equivalent to the absence of dissipation. In the remaining part of this section we find the velocity and temperature distributions in the presence of dissipation. For this, we substitute eqns (3.16) and (3.17) in eqns (3.08) and (3.09) and obtain coupled equations

$$
\begin{gathered}
\frac{d \phi}{d Y^{2}}-\sigma^{2} \phi+\epsilon=0 \\
\frac{d^{2} \epsilon}{d Y^{2}}+\left(\frac{d U_{0}}{d Y}\right)^{2}+\sigma^{2} U_{0}^{2}=0
\end{gathered}
$$




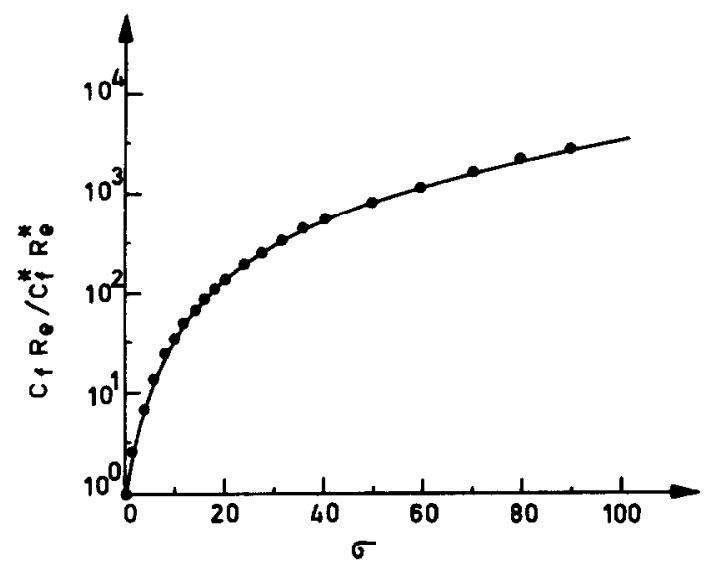

Fig. 4. Ratio of the product of friction factor and Reynold's number with and without the porous medium vs $\sigma$.

and the corresponding boundary conditions are

$$
\begin{aligned}
\phi=0 & \text { and } \epsilon=0 \text { at } Y=1 \\
\frac{\mathrm{d} \phi}{\mathrm{d} Y}=0 \text { and } \frac{\mathrm{d} \epsilon}{\mathrm{d} Y}=0 \text { at } & Y=0 .
\end{aligned}
$$

Solving eqns (3.32) and (3.33) satisfying conditions (3.34) and (3.35) we obtain

$$
\begin{array}{r}
\phi=\left[\left\{\frac{1}{2 \sigma^{4}}-\frac{3}{\sigma^{6}}+\frac{\cosh 2 \sigma}{4 \sigma^{6} \cosh ^{2} \sigma}\right\}-\frac{Y^{2}}{2 \sigma^{4}}+\frac{\cosh 2 \sigma Y}{12 \sigma^{2} \cosh ^{2} \sigma}+\frac{\cosh \sigma Y}{\cosh \sigma}\left\{\frac{3}{\sigma^{6}}+\frac{\tanh \sigma}{\sigma^{2}}-\frac{\cosh 2 \sigma}{3 \sigma^{6} \cosh ^{2} \sigma}\right\}\right. \\
\left.-\frac{Y \sinh \sigma Y}{\sigma^{5} \cosh \sigma}\right] \\
\epsilon=\left[\left\{\frac{1}{2 \sigma^{2}}-\frac{2}{\sigma^{4}}+\frac{\cosh 2 \sigma}{4 \sigma^{4} \cosh ^{2} \sigma}\right\}-\frac{Y^{2}}{2 \sigma^{2}}-\frac{\cosh 2 \sigma Y}{4 \sigma^{2} \cosh ^{2} \sigma}+\frac{2 \cosh \sigma Y}{\sigma^{4} \cosh \sigma}\right]
\end{array}
$$

Equations (3.36) and (3.37) tend to the perturbed velocity $\phi_{f}$ and temperature $\epsilon_{f}$ distributions in the case of flow through a channel without the porous material, in the limit $\sigma \rightarrow 0$ and they are of the form

$$
\phi_{f}=\frac{1}{360}\left[Y^{6}-15 Y^{2}+14\right]
$$

and

$$
\epsilon_{f}=\frac{1}{12}\left[1-Y^{4}\right]
$$

Equations (3.36) and (3.37) are numerically evaluated for different values of $\sigma$ and are shown in Figs. 5 and 6 respectively. In Fig. 7 the values of $\phi$ and $\epsilon$ on the centre-line ( $Y=0)$ are plotted against $\sigma$. The interesting feature of these results is that both $\phi$ and $\epsilon$ decrease rather rapidly with increasing $\sigma$ ( $\phi$ decreasing more rapidly than $\epsilon$ ), indicating that the decrease in permeability acts to decrease the effect of dissipation heating on the temperature and velocity profiles. As an example, when $\sigma$ is increased from 0 to $4, \phi$ decreases by $96.4 \%$ and $\epsilon$ by $69.2 \%$, and for higher values of $\sigma, \phi$ and $\epsilon$ are negligible. In other words, any increase in Darcy dissipation accompanying a decrease in permeability is more than overcome by the corresponding decrease in viscous dissipation. This decrease in viscous dissipation is due to the fact that $\sigma$ tends to flatten the velocity profile as well as decrease the flow. To know qualitatively the percentage of decrease in velocity and temperature distribution due to permeability the ratios $\phi / \phi_{f}$ and $\epsilon / \epsilon_{f}$ are numerically evaluated and are shown in Fig. 8. We find that they decrease very rapidly with increasing $\sigma$. 


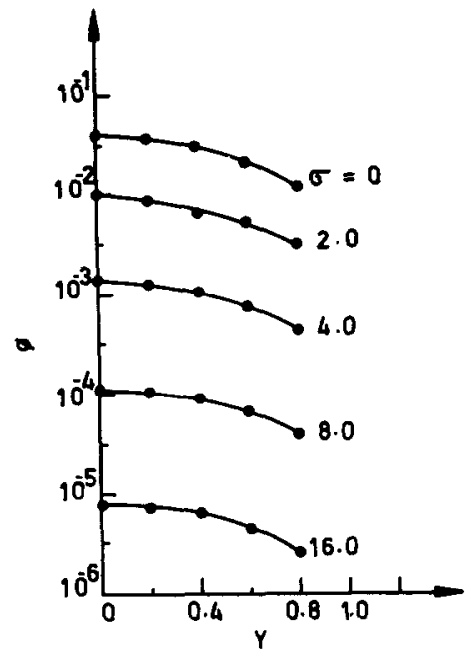

Fig. 5. Velocity correction profiles for various $\sigma$.

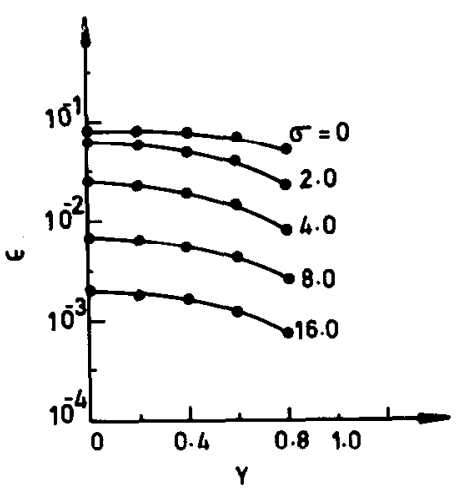

Fig. 6. Temperature correction profiles for various $\sigma$.

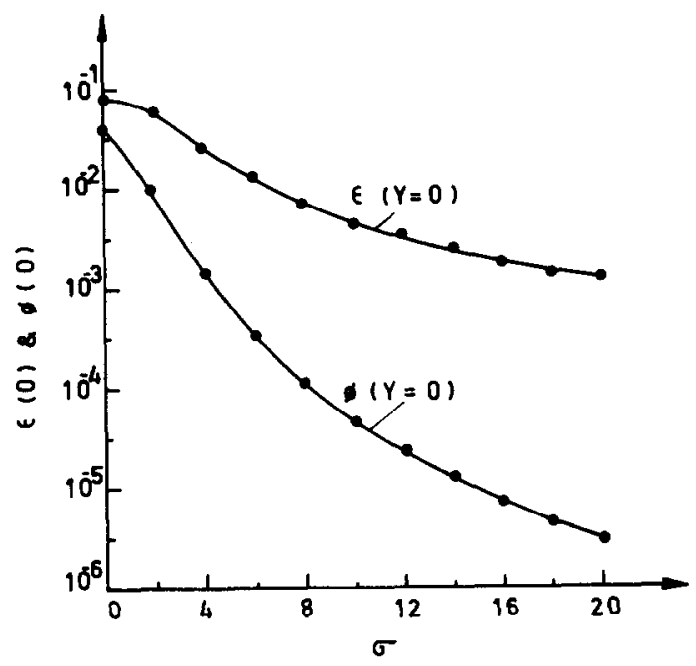

Fig. 7. Mid-channel velocity and temperature corrections for various $\sigma$.

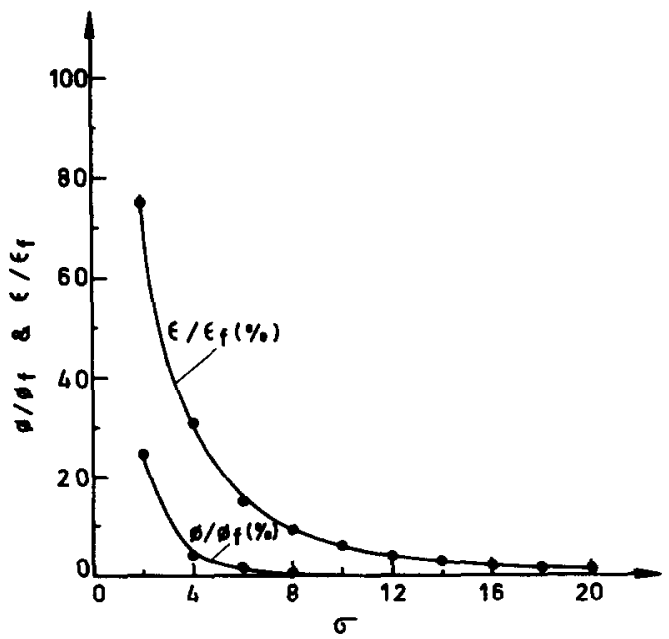

Fig. 8. Ratio of velocity and temperature corrections for flow with and without the porous medium vs $\sigma$. 
It is of practical interest to find the percentage increase or decrease in velocity distribution due to permeability of the material. Therefore the ratio of $\phi / U_{0}$ against $\sigma$ is shown in Fig. 9 . From this we conclude that the effect of buoyancy on the flow is negligible for large values of $\sigma$. The percentage increase or decrease in temperature distribution due to dissipation effects are shown in Fig. 10. As in the case of velocity distribution, here also we find that the effect of buoyancy on the temperature is negligible for large $\sigma$.

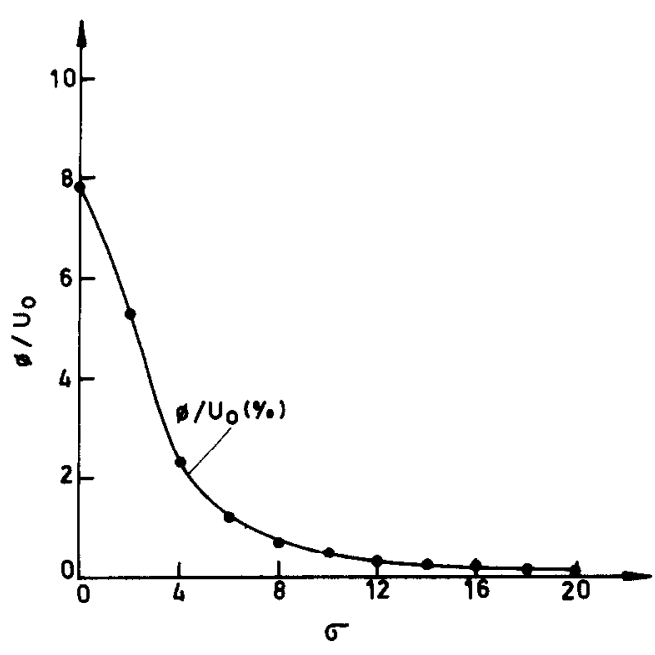

Fig. 9.

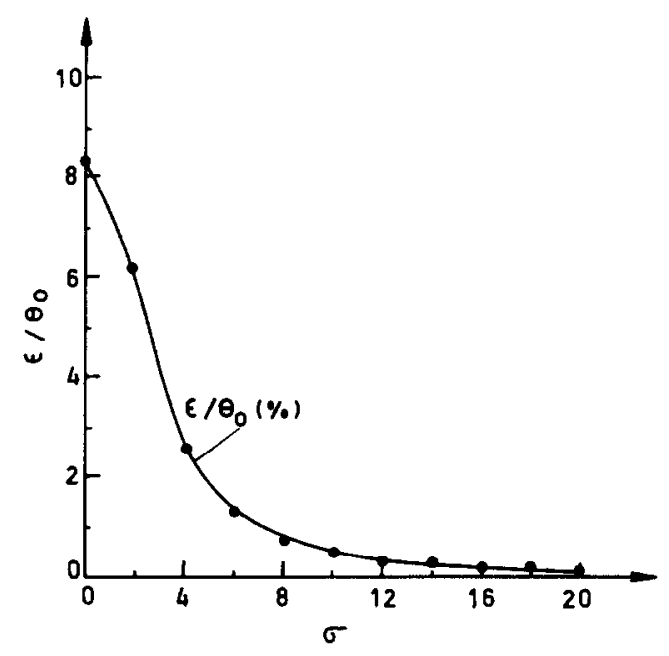

Fig. 10.

Fig. 9. Percentage ratio of velocity correction to velocity vs $\sigma$.

Fig. 10. Percentage ratio of temperature correction to temperature vs $\sigma$.

Knowing the velocity field, we can now calculate the skin friction, which is given by

$$
\tau^{\prime}=\mu\left(\frac{\partial \mu}{\partial y}\right)_{y=b}
$$

which in the non-dimensional form can be written, by using eqns (3.5) and (3.7), as

$$
\begin{aligned}
\mathrm{T} & =\frac{\tau^{\prime}}{\rho g \beta\left(T_{1}-T_{0}\right)} \\
& =\left(\frac{\mathrm{d} U}{\mathrm{~d} Y}\right)_{Y=1} .
\end{aligned}
$$

Hence from eqn (3.16) using eqns (3.18) and (3.36) we obtain

$$
\mathrm{T}=-\frac{\tanh \sigma}{\sigma}+N\left[-\frac{2}{\sigma^{4}}+\frac{5 \tanh \sigma}{3 \sigma^{5}}+\frac{\tanh \sigma}{3 \sigma^{5} \cosh ^{2} \sigma}+\frac{\tanh ^{2} \sigma}{\sigma^{4}}\right] .
$$

T is plotted against $\sigma$ in Fig. 11 for $N=+0.01$. The skin friction is practically the same for all values of $N$ and it decreases numerically with increasing $\sigma$.

It is of practical interest and importance to calculate the rate of heat transfer between the fluid and the plate. This is given by

$$
q^{\prime}=-K\left(\frac{\partial T}{\partial y}\right)_{y=b}
$$

which using eqns (3.5) and (3.6) takes the form

$$
\begin{aligned}
q & =-\frac{q^{\prime} b}{K\left(T_{1}-T_{0}\right)} \\
& =\left(\frac{\mathrm{d} \theta}{\mathrm{d} Y}\right)_{Y=1}
\end{aligned}
$$


Hence, from eqns (3.17) and (3.44) we get

$$
q=-\frac{N}{\sigma^{2}}\left[1-\frac{\tanh \sigma}{\sigma}\right]
$$

$q$ is shown in Fig. 12 for different values of $\sigma$ and $N=+0.01$. We observe from the figure that as $\sigma$ increases $q$ decreases. Also it can be seen from eqn (3.47) that any increase in $N$ causes an increase in $q$ by the same factor. Physically, this signifies that when $N>0$ the heat transfer is from the plate to the fluid while the opposite is true for $N<0$, i.e. the heat transfer and the nature of $N$ are oppositely directed.

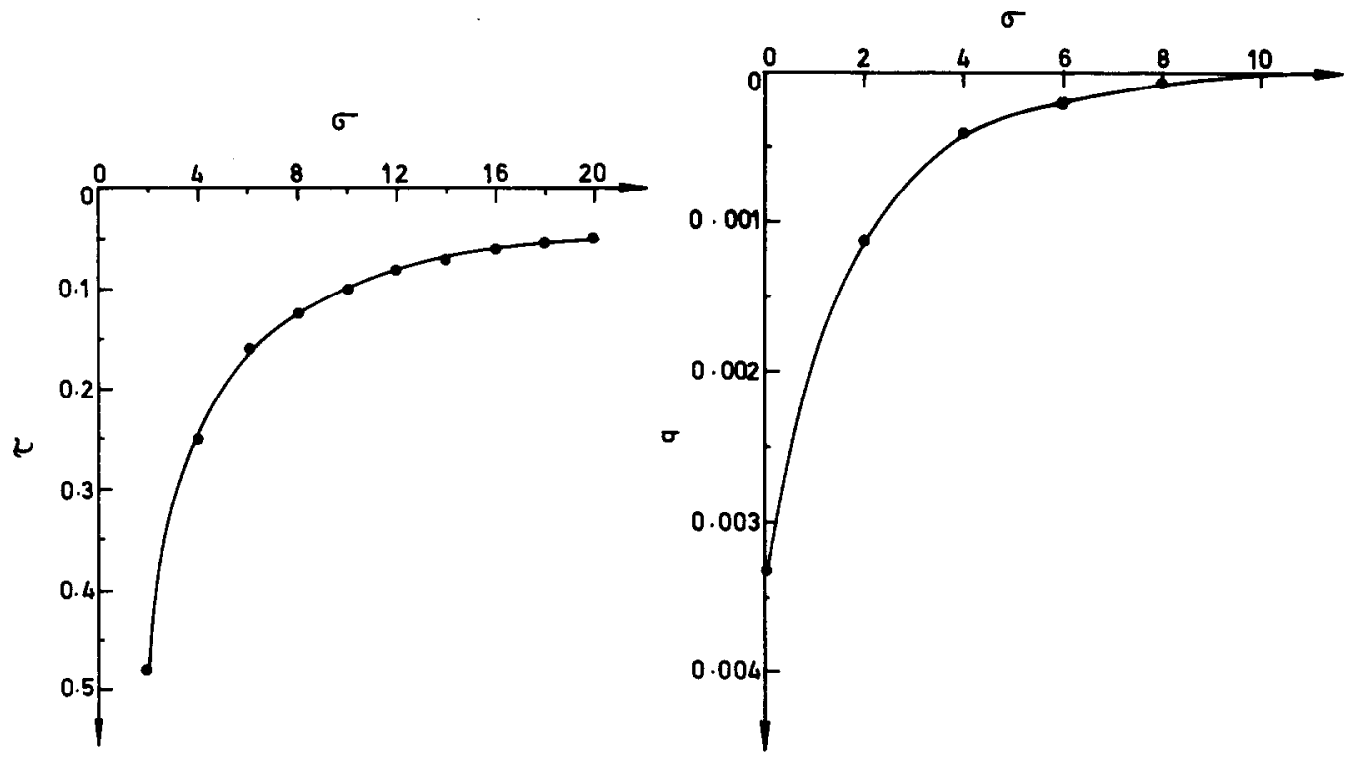

Fig. 11. Skin friction vs $\sigma$.

Fig. 12. Heat transfer vs $\sigma$.

\section{CONCLUSIONS}

The effect of free convection on the vertical flow through porous material is investigated and it is shown that when both viscous and Darcy resistances are included the coupled velocity and temperature equations are nonlinear. These non-linear equations are solved by a regular perturbation method with buoyancy force $N$ as a perturbation parameter and the following conclusions on velocity and temperature distributions are drawn.

(a) For $N>0$, eqns (3.16) and (3.17) show that the effect of buoyancy is to increase the velocity and temperature by greater heat addition due to viscous and Darcy dissipations or by increased cooling of the plates. However, the velocity and temperature are decreased considerably by an increase in the porous parameter $\sigma$. This effect of retardation due to $\sigma$ is useful for studying the pore size of a porous medium in which the magnitude of retardation is related to the permeability of the porous material.

For $N<0$, although the velocity and temperature are decreased, separation may be avoided for large $\sigma$.

In particular, we conclude that the perturbations on velocity and temperature produced by the buoyancy die out for large $\sigma$. Therefore, the flow may be laminar for large values of $\sigma$.

(b) The skin friction decreases with increasing $\sigma$ and practically remains the same for all values of $N$.

(c) The rate of heat transfer $q$ decreases with increasing $\sigma$. Any increase in $N$ causes an increase in $q$ by the same factor.

Acknowledgement-One of the authors (S.T.N.) is grateful to Dr. R. Narayana, Director of Instruction, College of Basic Sciences \& Humanities, University of Agricultural Sciences, Hebbal, Bangalore, India, for his encouragement and also to the University Grants Commission, India, for awarding Teacher Fellowship, vide order No. 8-117/76 (FIP). 
[2] C. K. W. TAM, J. Fluid Mech. 38, 537 (1969).

[3] R. PATIL PRABHAMANI and N. RUDRAIAH, Trans. ASME, J. Appl. Mech. 40 (4), 879 (1973).

[4] H. C. BRINKMAN, Appl. Sci. Res. A1, 27 (1947).

[5] H. LAMB, Hydrodynamics. Dover, New York (1945).

[6] E. M. SPARROW, G. S. BEAVERS and J. I. GREGG, Physics of Fluids 2, 319 (1959).

[7] S. OSTRACH, Nat. Adv. Comm. Aero. Note No. 2863 Washington (1952).

(Received 8 June 1976) 